\title{
Initial Clinical Efficacy and Safety of Microporous Polysaccharide Hemospheres (MPH) for Needle-Hole Bleeding from Expanded Polytetrafluoroethylene Grafts in Peripheral Vascular Surgery
}

\section{Furukawa $\mathrm{H}^{\star}$}

Department of Cardiovascular Surgery, Cardiovascular Center, Okayama Central Hospital, Okayama, Japan

\begin{abstract}
Background: In this retrospective study, the initial clinical effects of microporous polysaccharide hemospheres $(\mathrm{MPH})$ in peripheral vascular surgery were investigated.

Methods: Forty patients who underwent peripheral vascular surgery using expanded polytetrafluoroethylene (ePTFE) grafts were included. Between May 2008 and June 2012, 26 patients (group A) were administered MPH (Arista $\left.\mathrm{AH}^{\circledast}\right) 1 \mathrm{~g}$, while 14 (group $\mathrm{B}$ ) were treated with fibrin sealant (Beriplast $\mathrm{P}^{\circledR}$ ) $1 \mathrm{ml}$ at anastomosis between the prosthesis and native vessel during surgery. Perioperative results and complications including early graft patency, wound problems and postoperative general co-morbidities were retrospectively evaluated in both groups.

Results: The numbers of anastomoses between native vessels and ePTFE grafts were $2.2 \pm 0.5$ in group $A$ and $2.6 \pm 1.2$ in group $B(p=0.14)$. The mean amount of intraprocedural blood loss was significantly lower in group $A: 28.8$ $\pm 24.5 \mathrm{ml}$ than in group B: $88.2 \pm 93.7 \mathrm{ml}(p<0.01)$. The patencies of ePTFE grafts in the acute phase were $96.2 \%$ in group $A$ and $85.7 \%$ in group $B(p=0.24)$.
\end{abstract}

Conclusion: The results of the present study indicated that MPH contributed to acceptable and similar early clinical and surgical outcomes with safety and efficacy for hemostasis to those of fibrin sealant during peripheral vascular surgery using ePTFE grafts.

Keywords: Vascular surgery; Hemostasis; Fibrin sealant; Anastomosis; Intraprocedural

\section{Introduction}

Topical hemostatic agents are widely used in peripheral vascular surgery (PVS) [1]. Although local hemostatic agents were evaluated in a previous surgical experimental study [2], their clinical efficacies and unmet needs are still debated and remain at various clinical settings. Hemostasis between vascular anastomosis is considered to be the most important factor affecting clinical outcomes following PVS, especially topical hemostasis at suture- or needle-hole bleeding from anastomosis [3]. Needle-hole bleeding from anastomosis using expanded polytetrafluoroethylene (ePTFE) grafts may be one of the main issues affecting hemostasis during surgery, even when surgeons perform complete anastomoses. The aggressive use of hemostatic agents such as fibrin sealant for needle-hole bleeding at ePTFE grafts has been suggested to lead to better early clinical outcomes following surgery [4-6].

A hemostatic agent, microporous polysaccharide hemospheres $(\mathrm{MPH})$, which are controlled-porosity spherical particles made from starch, was introduced clinically from 2008 in Japanese clinical settings. The hemostatic effects of MPH have been demonstrated under various experimental settings [7-13]. Although the clinical effects of MPH have been reported in other fields such as neurosurgery, the clinical effects of MPH in cardiovascular surgery is unclear [14-18]. Therefore, the initial clinical efficacy of the hemostatic agent, MPH, in PVS was herein investigated.

\section{Patients and Methods}

Forty patients who underwent PVS using expanded polytetrafluoroethylene (ePTFE) prosthetic grafts were included in this study. Between May 2008 and June 2012, 26 patients (group A) were administered $\mathrm{MPH}$ (Arista $\mathrm{AH}^{\circledR}$, Medafor Inc., Minneapolis, MN) $1 \mathrm{~g}$ while 14 (group B) were treated with fibrin sealant (Beriplast $\mathrm{P}^{\circledR}$, CSL Behring, Marburg, Germany) $1 \mathrm{ml}$ at anastomosis between the prosthesis and native vessel during surgery. The preoperative characteristics of patients were shown in Table 1 . In group A, 10 patients exhibited lower extremity revascularization while 16 patients showed vascular access (VA). In group B, 9 patients exhibited peripheral arterial revascularization (PAR) and 5 had arteriovenous grafts (AVGs) $(\mathrm{p}=0.12)$. Preoperative anticoagulant agents were not stopped prior to the surgical intervention in any case. Perioperative results and complications including graft patency, wound problems, and postoperative general co-morbidities were retrospectively evaluated in both groups. I directly evaluated postoperative complications such as wound problems and graft patency was assessed by postoperative lower extremities enhanced multi-detector computed tomography or angiography for PAR patients. In AVG patients, clinical availability for VA was defined as graft patency.

Intraprocedural bleeding was estimated by vacuum and gauze

${ }^{*}$ Corresponding author: Furukawa $\mathrm{H}$, Department of Cardiovascular Surgery, Kawasaki Medical School, Kurashiki, Japan, 577 Matsushima Kurashiki-city, Okayama, 701-0192, Japan, Tel: +81-86-462-1111; Fax: +81-86-464-1189; E-mail: hfurukawa@med.kawasaki-m.ac.jp

Received September 09, 2016; Accepted September 14, 2016; Published September 20, 2016

Citation: Furukawa H (2016) Initial Clinical Efficacy and Safety of Microporous Polysaccharide Hemospheres (MPH) for Needle-Hole Bleeding from Expanded Polytetrafluoroethylene Grafts in Peripheral Vascular Surgery. J Vasc Med Surg 4 285. doi: 10.4172/2329-6925.1000285

Copyright: @ 2016 Furukawa H. This is an open-access article distributed under the terms of the Creative Commons Attribution License, which permits unrestricted use, distribution, and reproduction in any medium, provided the original author and source are credited. 
Citation: Furukawa H (2016) Initial Clinical Efficacy and Safety of Microporous Polysaccharide Hemospheres (MPH) for Needle-Hole Bleeding from Expanded Polytetrafluoroethylene Grafts in Peripheral Vascular Surgery. J Vasc Med Surg 4: 285. doi: 10.4172/2329-6925.1000285

Page 2 of 5

\begin{tabular}{|l|c|c|c|}
\hline & Group A & Group B & p-value \\
\hline No of patients & 26 & 14 & \\
\hline Mean age (years, \pm S.D.) & $73.0 \pm 9.9$ & $82.1 \pm 6.0$ & $<0.01$ \\
\hline Gender (female/male) & F:9, M:17 & F:4, M:10 & 0.7 \\
\hline Preoperative co-morbidities & & & \\
\hline HT & 21 & 8 & 0.12 \\
\hline DM & 7 & 5 & 0.57 \\
\hline DL & 7 & 1 & 0.14 \\
\hline CAD & 7 & 3 & 0.71 \\
\hline CVD & 2 & 5 & $<0.05$ \\
\hline CRF on HD & 17 & 6 & 0.17 \\
\hline Preoperative anticoagulant drug & 9 & 9 & 0.076 \\
\hline aspirin & 6 & 7 & 0.087 \\
\hline warfarin & 1 & 3 & 0.081 \\
\hline other anti-platelet agents & 8 & 3 & 0.53 \\
\hline
\end{tabular}

treatment was only considered if patients had consistent infectious or septic problems prior to surgery.

\section{Results}

Surgical outcomes were shown in Table 2. All patients were discharged, except for one in group B who died from respiratory failure due to aspiration pneumonia 31 days after surgery; therefore, surgical mortality was $0 \%$ in both groups, and hospital mortality was $0 \%$ in group A and $7.1 \%$ in group B ( $\mathrm{p}=0.17)$. The number of anastomoses between native vessels and ePTFE grafts was slightly higher in group B (2.6 \pm 1.2$)$ than in group A $(2.2 \pm 0.5)(\mathrm{p}=0.14)$. The mean amount of bleeding during surgery was significantly less in group A $(28.8 \pm 24.5$ $\mathrm{ml})$ than in group $B(88.2 \pm 93.7 \mathrm{ml})(\mathrm{p}<0.01)$. Reoperation for bleeding was performed for 1 patient in group $A$, in whom bleeding was not from anastomosis, and for no patients in group B. Postoperative complications and co-morbidities in both groups were shown in Table 3. In group A, non-infective wound edema was detected in 4 cases, the formation of seroma in 2, and reversible mild liver dysfunction caused by the adverse effects of postoperative prophylactic antibiotics in one. In group B, fatal aspiration pneumonia developed in one patient that resulted in hospital death, non-infective wound edema in 4 , and the deterioration of renal dysfunction in one. There was no wound infection in either group. The patencies of ePTFE grafts in the acute phase were $96.2 \%$ in group A and $85.7 \%$ in group B $(\mathrm{p}=0.24)$. No pseudoaneurysm was observed at the anastomosis site in the early phase following surgery.

\section{Discussion}

Hemostasis from anastomosis bleeding is a major and important issue for successfully performing surgery. Residual and consistent

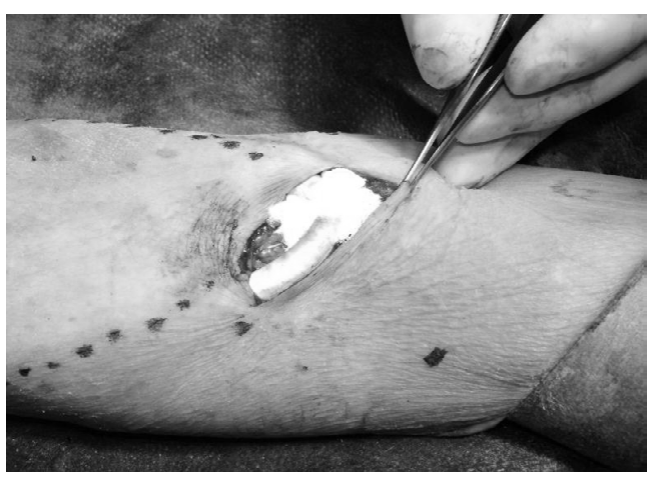

Figure 1: Intra-operative findings Microporous polysaccharide hemospheres (MPH) were administered at anastomosis of the forearm arteriovenous loop graft using an expanded polytetrafluoroethylene (ePTFE) Advanta ${ }^{\circledR}$ graft. vascular grafts, the loop graft was the most common configuration and loop AVGs at the forearm or thigh were created from the artery to the vein using local hemostatic agents at both anastomosis sites. Surgical sutures between native vessels and ePTFE vascular grafts were the same as those used in PVS. The ePTFE grafts were Distaflo ${ }^{\circledR}$ (Bard Peripheral Vascular Inc., Tempe, AZ, USA) in peripheral arterial surgery and Advanta $^{\oplus}$ (Atrium Medical, Hudson, NH, USA) in VA surgery (Figure 1). When the procedure was completed, all bleeding points had to be controlled. Hemostasis was defined as no visible bleeding at the suture line. After complete hemostasis, local antibiotics (Gentamicin 40 $\mathrm{mg}$ ) were routinely administered to prevent local infection following surgery, and surgeries were subsequently performed without any problems. Systemic heparinization was not reversed by a protamine injection in any case in both groups. Postoperative antibiotic strategies were basically employed for $48 \sim 72$ hours, and a prolonged antibiotic

\begin{tabular}{|l|c|c|c|}
\hline Anesthesia & Group A & Group B & p-value \\
\hline General & 10 & 10 & $<0.05$ \\
\hline Local & 16 & 4 & $<0.05$ \\
\hline Surgical mortality (within 30 days) & 0 & 0 & - \\
\hline Hospital mortality & 0 & 1 & 0.17 \\
\hline Mean number of anastomosis & $2.2 \pm 0.5$ & $2.6 \pm 1.2$ & 0.14 \\
\hline Intraprocedural blood loss (ml) & $28.8 \pm 24.5$ & $88.2 \pm 93.7$ & $<0.01$ \\
\hline Intraprocedural transfusion (units) & 0 & 0 & - \\
\hline Early reoperation for bleeding & 1 & 0 & 0.46 \\
\hline Patency of prosthetic ePTFE grafts & 25 & 12 & 0.24 \\
\hline Pseudoaneurysm & 0 & 0 & - \\
\hline
\end{tabular}

RBC: Red blood cell, ePTFE grafts: Expanded Polytetrafluoroethylene Grafts. Table 2: Surgical outcomes. 
Citation: Furukawa H (2016) Initial Clinical Efficacy and Safety of Microporous Polysaccharide Hemospheres (MPH) for Needle-Hole Bleeding from Expanded Polytetrafluoroethylene Grafts in Peripheral Vascular Surgery. J Vasc Med Surg 4: 285. doi: 10.4172/2329-6925.1000285

Page 3 of 5

\begin{tabular}{|l|c|c|c|}
\hline & Group A & Group B & p-value \\
\hline Postoperative co-morbidities & & & \\
\hline Seroma & 2 & 0 & 0.29 \\
\hline Wound dehiscence & 0 & 0 & - \\
\hline Wound infection & 0 & 0 & - \\
\hline Persistent fever & 0 & 0 & - \\
\hline New-onset CVD & 0 & 0 & - \\
\hline Liver dysfunction & 1 & 0 & 0.46 \\
\hline Deterioration of CRF & 0 & 1 & 0.17 \\
\hline Respiratory disorder & 0 & 1 & 0.17 \\
\hline MACE & 0 & 0 & - \\
\hline Thrombosis in the acute phase & 1 & 2 & 0.24 \\
\hline Postoperative transfusion & 2 & 5 & $<0.05$ \\
\hline Local swelling & 4 & 4 & 0.33 \\
\hline Uncontrolled pain & 1 & 1 & 0.65 \\
\hline Postoperative discharge & 1 & 0 & 0.46 \\
\hline
\end{tabular}

CVD: Cerebrovascular disease, CRF Chronic renal failure, MACE: Major adverse cardiac events.

"Persistent fever: fever that continued for more than one week following surgery.

Table 3: Postoperative co-morbidities.

bleeding from anastomosis in PVS may affect surgical and clinical outcomes. Intraoperative bleeding is exacerbated by the intraoperative use of heparin, which is routinely administered in vascular surgery to prevent intra- and postoperative thrombosis. The need for local hemostatic agents in PVS has been proposed by various groups. Local hemostatic agents are used in PVS because vascular surgeons continue to be confronted with the difficulties associated with treating increasingly elderly patients. As the elderly patients who underwent PVS had diseased arteries, therefore, local hemostatic agents at anastomosis may help to achieve complete hemostasis. All hemostatic agents have clinical advantages and shortcomings; however, objective comparative data between modern topical hemostatic agents in a clinical vascular surgical setting are still limited. Clinical trial settings are difficult to perform because the cause of bleeding is diverse; the origin of bleeding may be arterial, venous, or oozing. In both groups, the prevalence of preoperative anticoagulation/antiplatelet therapy differed, and blood pressure variation may affect the perioperative bleeding tendency. All these factors may influence bleeding and affect the interpretation of clinical data. Previous studies addressed the clinical role and efficacies of local hemostatic agents. In an experimental study, CoSeal ${ }^{\circ}$, a local hemostatic agent, was shown to significantly reduce the time to hemostasis and blood loss [19]. In 164 vascular patients who underwent either a peripheral arterial bypass or AVG procedure, rThrombin had a superior immunogenicity profile to those of other hemostatic agents without specific adverse events [20].

ePTFE vascular grafts have been introduced and adopted because of their clinical benefits such as their low thromobogenicity, porosity, and limited elasticity. However, suture-line bleeding at vascular anastomoses increases operating times. Bleeding is regarded as an unavoidable and inevitable consequence of joining an ePTFE graft to an artery. Suture-hole bleeding is typically managed by manual compression with surgical swabs and the reversal of heparin, which is still the generally accepted standard of care. ePTFE graft placement may be complicated by prolonged anastomotic suture-hole bleeding, which increases operative times, overall blood loss, and the risk of wound complications. Vascular surgeons encounter oozing bleeding from the suture-line of ePTFE vascular grafts, which sometimes becomes an uncontrollable clinical issue. Therefore, the routine use of a local hemostatic agent in vascular surgery using ePTFE grafts has been recommended as a cost- and time-saving strategy.

FS is a representative hemostatic agent that has been widely used in various clinical settings. FS contains the components necessary for the generation of a fibrin clot and has the ability to achieve hemostasis or sealing independently of the patient's coagulation system. FS is beneficial for the time required for hemostasis and blood loss in the ePTFE vascular graft suture-line. Previous studies clearly demonstrated that the differences between products with respect to manufacturing processes, compositions, and packaging affected their ease of and indications for use, storage, and handling, as well as their safety profiles. The use of FS is beneficial for the treatment of challenging suture-line bleeding at vascular anastomoses [4]. The present larger randomized, prospective, multicenter study was initiated to evaluate the efficacy and safety of a new investigational form of FS in a clinical setting [1]. FS achieved more rapid hemostasis than traditional techniques in upper extremity VA surgery using ePTFE graft placement for dialysis. Surgery is expensive; therefore, an effective, rapidly performing hemostatic agent may not only enhance patient care, but also reduce costs [5]. Schenk et al. reported that FS was safe and its efficacy was superior to manual compression for hemostasis in patients with peripheral vascular ePTFE grafts and VA surgery [17]. A comparison with a thrombin-soaked gelatin sponge (TSG) for its efficacy in stopping from needle- or suture-hole bleeding in ePTFE grafts after anastomosis to the femoral artery of 235 subjects indicated that FS was more effective than TSG for achieving hemostasis [6]. A cyanoacrylate surgical sealant is also effective at reducing the time to hemostasis and achieving immediate hemostasis in arteriovenous shunt and femoral bypass procedures and is considered safe for internal use, as reported by Lumsden and colleagues [21]. Thus, an ideal hemostatic agent has not yet been established in peripheral vascular surgery.

MPH comprise plant-based polysaccharides that are synthesized into spherical microporous particles and are highly effective at achieving topical hemostasis. The fine, dry, sterilized white powder only contains plant-based products with no animal or human components. The powder consists of hydrophilic microporous particles synthesized by purified potato starch through a proprietary process. $\mathrm{MPH}$ are biocompatible and nonpyrogenic, and are considered to be absorbed within 24 to 48 hours. Their mechanism of action involves platelets, red blood cells, and blood proteins being concentrated on the particle surfaces to form a gelled matrix after fluid is absorbed. This gel enhances normal clotting reactions and creates stable hemostatic plugs. The particles act as molecular sieves that dehydrate blood while concentrating individual components on their surface and accelerating the natural clotting cascade. MPH accelerate the natural clotting cascade through this unique mechanism by concentrating clotting factors and proteins on their surface, and, thus, exhibit hemostatic effects.

In a previous experimental study, MPH were found to be a highly effective hemostatic agent in the treatment of prolonged access bleeding [7]. MPH also provided a rapid and effective means of hemostasis for laparoscopic renal parenchymal injuries [11]. MPH was previously reported to achieve rapid, effective, and durable hemostasis in a porcine open partial nephrectomy model [10], and the findings of an initial study using an experimental porcine model suggested that $\mathrm{MPH}$ provided effective parenchymal hemostasis during laparoscopic resection of a kidney lesion [9]. Topical hemostatic agents are also widely used in brain surgery, but have some disadvantages such as foreign body reactions, being a focus for infection, and causing artifacts in radiological examinations. The most important advantage of MPH is their rapid clearance and absorption from the surgical field, thereby reducing the potential of imaging artifacts [12]. Residual topical 
Citation: Furukawa H (2016) Initial Clinical Efficacy and Safety of Microporous Polysaccharide Hemospheres (MPH) for Needle-Hole Bleeding from Expanded Polytetrafluoroethylene Grafts in Peripheral Vascular Surgery. J Vasc Med Surg 4: 285. doi: 10.4172/2329-6925.1000285

Page 4 of 5

hemostatic materials may serve as a nidus for infection or enhance infection in an already contaminated wound [8]. In a comparative experimental study between MPH and another hemostatic agent, FloSeal $^{\bullet}$, MPH did not affect or have negative effects on the healing of sinus mucosa [13]. Thus, the ideal hemostat does not require unusual storage or preparation, provides durable hemostasis, is degraded rapidly, and does not enhance surgical site infection.

The use of MPH as a local hemostatic agent has increased in clinical settings in the last decade. No intraoperative complications, including allergic reactions or decreases or increases in blood pressure or heart rate, were reported in previous neurosurgical studies. Thus, the topical application of MPH appears to be feasible for achieving sufficient hemostasis in neurosurgical procedures [14]. The use of MPH after endoscopic sinus surgery (ESS) was shown to result in significantly less bleeding in the early postoperative period with no increase in pain, obstruction, or nasal discharge [15]. However, a previous study reported that MPH increased the incidence of bleeding through dressing over that through electrocautery, but did not increase the incidence of active bleeding upon dressing removal [16]. The efficacy of MPH may be increased by the addition of a fibrinolytic inhibitor such as tranexamic acid or crystallized calcium ions to the MPH powder because physiological coagulation requires the presence of calcium [2]. Matsushita, et al. reported a unique and novel hemostatic combination with two plant-based hemostatic agents using MPH and oxidized regenerated cellulose [18].

To the best of our knowledge, this is the first clinical evidence to show the clinical efficacy of MPH in vascular surgery. Furthermore, the clinical effects of MPH in PVS have not yet been compared with those of other local hemostatic agents. In this present study, the aged population was significantly higher, and preoperative anticoagulant therapy was slightly more prevalent. This bias may have affected the significant increase observed in intraprocedural blood loss in group B. Further studies are needed to further our understanding of the clinical use of $\mathrm{MPH}$ in vascular surgery. As described previously, our vascular surgeons face difficulties with vascular anastomosis between diseased vessels in increasingly elderly patients; therefore, effective and cost-saving local hemostatic agents must be adopted and used more frequently in clinical settings.

The authors were solely responsible for the design and conduct of this study, all study analyses, the drafting and editing of the manuscript, and its final contents. Although the number of patients in the present study was small, we attempted to provide a clear strategy for tackling a difficult and unusual entity.

\section{Study Limitations}

There were several limitations to the present study. We conducted a single-center, retrospective, non-randomized study. Furthermore, the sample size was too small to obtain significant evidence and the clinical efficacy of these surgical techniques. Mid-term and long-term clinical evaluations are currently being performed. Further studies need to include a larger sample size and multiple institutions. There was a potential selection bias for patient inclusion in the study group, even among consecutive patients. Therefore, the accumulation of prospective data is needed to prevent such selection bias. A propensitymatched analysis may be mandatory to precisely evaluate clinical evidence.

\section{Conclusion}

The results of the present study indicated that MPH contributed to acceptable and similar early clinical and surgical outcomes with safety and efficacy for hemostasis during PVS using ePTFE grafts. Further studies are needed in order to elucidate the clinical effects of MPH in more detail.

\section{References}

1. Schenk III WG, Burks SG, Gagne PJ, Kagan SA, Lawson JH, et al. (2003) Fibrin sealant improves hemostasis in peripheral vascular surgery: A randomized prospective trial. Ann Surg 237: 871-876.

2. Björses K, Holst J (2007) Various local hemostatic agents with different modes of action; an in vivo comparative randomized vascular surgical experimental study. Eur J Vasc Endovasc Surg 33: 363-370.

3. Unlü Y, Vural U, Kocak H, Ceviz M, Becit N, et al. (2002) Comparison of the topical haemostatic agents for the prevention of suture hole bleeding. An experimental study. Eur J Vasc Endovasc Surg 23: 441-4.

4. Saha SP, Muluk S, Schenk III W, Burks SG, Grigorian A, et al. (2011) Use of fibrin sealant as a hemostatic agent in expanded polytetrafluoroethylene graft placement surgery. Ann Vasc Surg 25: 813-822.

5. Saha SP, Muluk S, Schenk W III, Dennis JW, Ploder B, et al. (2012) A prospective randomized study comparing fibrin sealant to manual compression for the treatment of anastomotic suture-hole bleeding in expanded polytetrafluoroethylene grafts. J Vasc Surg 56: 134-341.

6. Taylor LM Jr, Mueller-Velten G, Koslow A, Hunter G, Naslund T, et al (2003) Prospective randomized multicenter trial of fibrin sealant versus thrombin-soaked gelatin sponge for suture- or needle-hole bleeding from polytetrafluoroethylene femoral artery grafts. J Vasc Surg 38: 766-771.

7. Humphreys MR, Castle EP, Andrews PE, Gettman MT, Ereth MH, et al. (2008) Microporous polysaccharide hemospheres for management of laparoscopic trocar injury to the spleen. Am J Surg 195: 99-103.

8. Ereth MH, Dong Y, Schrader LM, Henderson NA, Agarwal S, et al. (2009) Microporous polysaccharide hemospheres do not enhance abdominal infection in a rat model compared with gelatin matrix. Surg Infect 10: 273-276.

9. Murat FJL, Le CQ, Ereth MH, Piedra MP, Dong Y, et al. (2006) Evaluation of microporous polysaccharide hemospheres for parenchymal hemostasis during laparoscopic partial nephrectomy in the porcine model. J Soc Laparoendoscopic Surg 10: 302-306

10. Murat FJL, Ereth MH, Dong Y, Piedra MP, Gettman MT, et al. (2004) Evaluation of microporous polysaccharide hemospheres as a novel hemostatic agent in open partial nephrectomy: Favorable experimental results in the porcine model. J Urol 172: 1119-22.

11. Humphreys MR, Lingeman JE, Terry C, Castle EP, Andrews PE, et al. (2008) Renal injury and the application of polysaccharide hemospheres: A laparoscopic experimental model. J Endourol 22: 1375-1382

12. Emmez H, Tonge M, Tohgoz N, Durdag E, Gonul I, et al. (2010) Radiologica and histopathological comparison of microporous polysaccharide hemospheres and oxidized regenerated cellulose in the rabbit brain: A study of efficacy and safety. Turkish Neurosurgery 20: 485-491.

13. Antisdel JL, Janney CG, Long JP, Sindwani R (2008) Hemostatic agent microporous polysaccharide hemospheres (MPH) does not affect healing or intact sinus mucosa. Laryngoscope 118: 1265-1269.

14. Tschan CA, Nie $\beta$ M, Schwandt E, Qertel J (2011) Safety and efficacy of microporous polysaccharide hemospheres in neurosurgery. Neurosurgery 69 : 49-63.

15. Antisdel JL, West-Denning JL, Sindwani R (2009) Effect of microporous polysaccharide hemospheres (MPH) on bleeding after endoscopic sinus surgery: Randomized controlled study. Otolaryngol Head Neck Surg 141: 353-357.

16. Tan SR, Tope WD (2004) Effectiveness of microporous polysaccharide hemospheres for achieving hemostasis in Mohs micrographic surgery Dermatol Surg 30: 908-914

17. Schenk WG, Goldthwaite CA Jr, Burks S, Spotnitz WD (2002) Fibrin sealan facilitates hemostasis in arteriovenous polytetrafluoroethylene grafts for renal dialysis access. Am Surg 68: 728-732.

18. Matsushita T, Masuda S, Inoue T (2010) Early experience with combined use of two plant-based hemostatic agents. Ann Thorac Surg 90: 327-328. 
Citation: Furukawa H (2016) Initial Clinical Efficacy and Safety of Microporous Polysaccharide Hemospheres (MPH) for Needle-Hole Bleeding from Expanded Polytetrafluoroethylene Grafts in Peripheral Vascular Surgery. J Vasc Med Surg 4: 285. doi: 10.4172/2329-6925.1000285

Page 5 of 5

19. Hill A, Estridge TD, Maroney M (2001) Treatment of suture line bleeding with a novel synthetic surgical sealant in a canine iliac PTFE graft model. J Biomed Mater Res 58: 308-312.

20. Weaver FA, Lew W, Granke K, Yonehiro L, Delange B, et al. (2008) A comparison of recombinant thrombin to bovine thrombin as a hemostatic ancillary in patients undergoing peripheral arterial bypass and arteriovenous graft procedures. J Vasc Surg 47: 1266-73.

21. Lumsden AB, Heyman ER (2006) Prospective randomized study evaluating an absorbable cyanoacrylate for use in vascular reconstructions. J Vasc Surg 44: $1002-1009$ 